\title{
Fatiga por contacto esférico en materiales multicapa de alúmina circona
}

\author{
E. JIMÉNEZ-PIQUÉ', L. CESERACCIU', M. ANGLADA', F. CHALVET², G. DE PORTU² \\ 'Departamento de Ciencia de Materiales e Ingeniería Metalúrgica, Universidad Politécnica de Cataluña, Avda. Diagonal 647 (ETSEIB), 08028 Barcelona \\ ${ }^{2}$ Istituto di Scienza e technologia dei materiali ceramici-CNR. Via Granolo 64, 48018 Faenza. Italia.
}

\begin{abstract}
Es usual que en aplicaciones reales los materiales cerámicos sufran fatiga por contacto. Una estrategia para mejorar su resistencia a la aparición de fatiga por contacto es la utilización de materiales con tensiones residuales de compresión en la superficie. En concreto, en este trabajo se presenta el estudio de la resistencia al contacto esférico estático y cíclico de un cerámico multicapa de alúmina y alúmina / circona producido por colaje en cinta, en el que aparecen tensiones residuales debido al desajuste entre los coeficientes de expansión térmica de las capas. También se estudia una alúmina monolítica con objeto de realizar comparaciones con un material sin tensiones residuales. Se muestra que la resistencia a la aparición de grieta anillo es mayor en el caso del material multicapa que en el de la alúmina, tanto en contacto estático como cíclico. También se muestra que existe fatiga mecánica en los dos materiales bajo contacto cíclico, y no sólo corrosión bajo tensión. Finalmente se propone una relación simple entre carga aplicada y tiempo hasta aparición de daño.
\end{abstract}

Palabras clave: Multicapas, Alúmina, Circona, Contacto Esférico, Fatiga, Corrosión Bajo Tensión.

\section{Hertzian fatigue in alumina/zirconia laminated composites}

In some engineering applications, ceramic materials are subjected to contact fatigue. In order to improve the resistance of ceramics to contact fatigue, it is proposed to use ceramic laminates with residual stresses at the surface. In this work, the Hertzian contact fatigue of an alumina-alumina/zirconia laminated composite produced by tape casting is studied, both under static loading and cyclic loading, together with a monolithic alumina for comparison purposes. It is shown that the laminate composite presents better resistance to the apparition of surface ring crack, due to the residual stresses of the laminate composite. It is also shown that there is a fatigue effect on both materials under cyclic loading, and not only stress corrosion cracking. A simple relationship between applied load and time to apparition of damage is finally proposed.

Keywords: Laminated composites. Alumina, Zirconia, Hertzian contact, Fatigue, Stress corrosion cracking

\section{INTRODUCCIÓN}

El uso de materiales cerámicos en aplicaciones reales se ve obstaculizado por su baja tenacidad y su gran dispersión en valores de resistencia mecánica, lo que compromete la integridad estructural de los componentes en servicio. Así, desde hace varias décadas se trabaja extensivamente en aumentar la tenacidad de las cerámicas, ya sea controlando la calidad de la microestructura, y por tanto, la población de defectos susceptibles de iniciar el fallo catastrófico, o haciendo que el material sea más resistente a la propagación de grietas una vez iniciadas, (mediante, por ejemplo transformaciones de fase), o fabricando composites cerámicos, ya sea con microestructuras dúplex, fibras o cerámicas multicapa. Estas cerámicas multicapa son una alternativa prometedora, ya que presentan valores altos de tenacidad y, en ciertos casos, la capacidad de frenar el crecimiento de grieta una vez iniciada ésta $[1,2,3]$.

La estrategia más utilizada en los materiales multicapa es la introducción de tensiones residuales [1], que se crean generalmente apilando capas de materiales con diferentes coeficientes de expansión térmica, de tal manera que, al bajar la temperatura desde la de sinterización y la de uso, se desarrollen tensiones residuales en las capas, ya sean de compresión o de tensión, debido a la diferencia en las dilataciones de las capas $[4,5,6]$. Uno de los multicapas más utilizados es el de alúmina / circona, debido a las excelentes propiedades de éstos materiales y a la buena unión que forman, aunque es usual utilizar un composite alúmina-circona en una de las capas para evitar excesivas tensiones residuales que produzcan agrietamiento en el material. Adicionalmente, la utilización de circona parcialmente estabilizada permite introducir tensiones residuales por transformación de fase t-m [7,8], y no sólo por diferencia de coeficientes de expansión térmica.

En estos materiales el aumento de tenacidad se consigue por dos razones principales. Primero, porque el factor de intensidad de tensiones de la grieta disminuye cuando se encuentra en la capa con tensión residual compresiva $(9,10)$ . Segundo, porque la grieta se desvía con un ángulo al llegar a la interfase entre los dos materiales debido a que existe un desajuste elástico entre materiales que provoca que el factor de intensidades de tensiones local tenga un modo mixto I/ II y, en menor medida, debido a las tensiones residuales [11, 12, 13].

Cuando estos laminados se diseñan de tal manera que una de las capas es suficientemente pequeña y con una alta tensión residual aparece el fenómeno de tensión umbral, 
investigado en profundidad por, entre otros, Rao y col. [14]. Estos materiales presentan un valor de resistencia mecánica independiente del tamaño de defecto (para un rango de defectos), es decir, la tensión de rotura es independiente de la entalla inicial, con lo que no habrá fallo catastrófico por debajo de una tensión umbral. La razón de este comportamiento es que la capa a compresión consigue frenar el crecimiento de la grieta. Por tanto estos materiales cerámicos presentan una insensibilización al defecto inicial con lo que el material no puede fracturarse por debajo de cierta tensión. [15, 16]. Este valor de tensión umbral puede aumentarse introduciendo grietas laterales (edge crack) en la capa de compresión, lo que provoca la bifurcación de la grieta, con el consiguiente aumento de energía de fractura. $[7,8,17,18,19]$.

Es frecuente que en servicio los materiales estén sometidos a contactos mecánicos, que degradan la superficie y pueden generar defectos que propaguen hasta el fallo catastrófico. Adicionalmente muchas veces estos contactos son cíclicos, con lo que aparecen efectos de fatiga. Con el objeto de mejorar las propiedades de contacto es posible utilizar materiales multicapa donde la capa superficial está a compresión, mejorando la tenacidad superficial aparente [20] y las propiedades tribológicas [21, 22].

Una de las metodologías más extendidas para el estudio de la mecánica de contacto es la indentación de los materiales con esferas, conocida como indentación Hertziana [23]. En contraste con la indentación puntiaguda, dónde existe deformación inelástica desde el primer contacto debida a la singularidad de la punta del indentador, la indentación Hertziana proporciona un contacto puramente elástico hasta una carga crítica, pudiéndose producir fractura sin apenas deformación, Además, la existencia de una solución analítica para el campo de deformación permite un análisis de la fractura más sencillo, ya que no hay fuertes tensiones residuales introducidas por deformación inelástica. El contacto de una esfera con la superficie de un material cerámico induce dos tipos principales de daño [24]: Para materiales con tenacidad muy baja, aparece una grieta cerca del radio de contacto, que adopta forma de cono al profundizar en el material. Este es un daño "clásico", que ya reportó Hertz en 1881. Sin embargo, para materiales cerámicos con tenacidad más elevada aparece daño "casi-plástico", debido al crecimiento y coalescencia de microgrietas situadas bajo el indentador por tensiones de cizalladura [25]. Cuando se indenta un bimaterial (por ejemplo un recubrimiento o una multicapa), aparecen otros tipos de daño, como delaminación o grietas radiales, dependiendo de las propiedades mecánicas de los materiales y de las dimensiones relativas de las capas del material y del indentador, tal y como demostraron Lawn y el grupo de Guiberteau, entre otros, para materiales modelo y materiales biomédicos [26, 27, 28, 29, 30]. Para el caso específico de materiales cerámicos multicapas, por ejemplo, An y col. combinaron una primera capa de $\mathrm{Al}_{2} \mathrm{O}_{3}$ (rotura frágil) con una segunda capa de $\mathrm{Al}_{2} \mathrm{O}_{3}: 30 \mathrm{Ca} \mathrm{Al}_{12} \mathrm{O}_{19}$ (rotura casi-plástica), consiguiendo un aumento de la resistencia mecánica del multicapa [31], y Jitcharoen y col. inhibieron el crecimiento de grieta hertziana utilizando un material con función gradiente de alúmina y vidrio de silicato de aluminio [32].

Así mismo, existen varios estudios de fatiga por contacto en materiales cerámicos [33], especialmente los efectuados por Lawn y col., tanto en materiales cerámicos frágiles [34] -donde atribuyen todos los efectos de fatiga a mecanismos corrosión bajo tensión-, como en materiales cerámicos casi-plásticos [35, 36], en los cuales se cuantifica el daño de fatiga producido en el material por el contacto midiendo la degradación de la resistencia mecánica. Fett y col. estudiaron la fatiga de alúmina por contacto cilíndrico (37), y mostraron que, para sus condiciones experimentales particulares, no existía corrosión bajo tensión bajo contacto estático, atribuyéndolo a que la grieta está cerrada en la punta (modo I negativo) con lo que el vapor de agua no puede penetrar (38). Así mismo, existen varios estudios en los que se efectúan ensayos de fatiga por contacto en nitruro de silicio observando la aparición de daño o no para un determinado conjunto de cargas y numero de ciclos $[39,40,41]$, así como estudios en vidrio en diferentes ambientes [42] y estudios de recubrimientos de vidrio bioactivo en aleaciones de titanio [43].

En este artículo se presenta el estudio de la fatiga por contacto esférico de un multicapas cerámico producido por colaje en cinta de alúmina y alúmina-circona, empleando tanto cargas cíclicas como estáticas. Este material multicapa tiene una capa superficial de alúmina de unas $200 \mu \mathrm{m}$ de espesor, y debido a que las cargas utilizadas y el radio del indentador son relativamente pequeños, la zona de daño está confinada a la primera capa. Además, debido al tamaño de su microestructura, el material estudiado presenta un comportamiento frágil, con lo que la grieta Hertziana es el primer daño que aparece. Para evaluar el daño producido por la indentación esférica, se efectúan inspecciones superficiales para rangos de carga aplicada y tiempo / número de ciclos. Éstos mismos ensayos se efectúan en una alúmina monolítica con similar microestructura.

\section{PROCEDIMIENTO EXPERIMENTAL}

\subsection{Preparación de los materiales}

Los materiales fueron producidos por colaje en cinta a partir de polvos de: (a) alúmina (Alcoa A16-SG, Alcoa Aluminium Co., Nueva York, EEUU) de alta pureza, 99.7\%, de tamaño medio de grano de $0.3 \mu \mathrm{m}$ y (b) Circona tetragonal policristalina (TZ3Y Tosho Corp. Japón), 94.7\% $\mathrm{ZrO}_{2}, 3 \mathrm{~mol} \%$ $\mathrm{Y}_{2} \mathrm{O}_{3}$ (3Y-TZP), de tamaño medio de grano de $0.3 \mu \mathrm{m}$. Los polvos fueron mezclados con defloculantes, dispersantes, plastificadores y disolventes orgánicos para obtener suspensiones adecuadas para colaje en cinta. La suspensión fue colaje en cinta en un sustrato de mylar desplazado a una velocidad de $200 \mathrm{~mm} / \mathrm{min}$ [44].

Se prepararon láminas de alúmina pura -designadas como "A"- y láminas de alumina-circona (60/40 en volumen) designadas como "AZ"- con espesores en verde seleccionados para obtener capas de $200 \mu \mathrm{m}$ (A) y $350 \mu \mathrm{m}$ (AZ) después de la sinterización. Después del secado, se cortaron láminas de $50 * 34 \mathrm{~mm}$ y se apilaron alternativamente (A-AZ-A-...-A) siete capas de $\mathrm{A}$ y seis de $\mathrm{AZ}$, sometiendo el multicapa resultante -designado como A/AZ- a una presión de $30 \mathrm{MPa}$ a $75{ }^{\circ} \mathrm{C}$ durante 30 minutos. A continuación se calentó hasta $600{ }^{\circ} \mathrm{C}$ a velocidades de calentamiento bajas, seguido de un sinterizado a $1550{ }^{\circ} \mathrm{C}$ durante una hora. Las muestras finales obtenidas después del sinterizado presentan una densidad del $97 \%$ y espesores de $3 \mathrm{~mm}$, con una relación entre espesores de capa de $1 / 1.3$ (siendo AZ la capa más gruesa).

Adicionalmente, se prepararon muestras de alúmina pura (designada MA) por presión isoestática en frío y sinterizado a $1550{ }^{\circ} \mathrm{C}$ durante una hora, con el objeto de obtener muestras sin tensiones residuales para comparación. 


\subsection{Caracterización mecánica}

Tanto las muestras A/ AZ como las MA fueron cortadas en barras prismáticas de $4^{*} 3^{*} 20 \mathrm{~mm}$, y la capa superior (alúmina) fue pulida con suspensión de diamante de hasta $3 \mu \mathrm{m}$. La microestructura fue observada con microscopía electrónica de barrido (MEB) en muestras atacadas térmicamente (1500 ${ }^{\circ} \mathrm{C}, 30$ minutos), observándose que el tamaño de grano de las capas de alúmina de A/AZ era comparable al de MA y aproximadamente igual a $1.5 \mu \mathrm{m}$. También se observó que la interfase de las diferentes capas de A/ AZ estaba bien unida.

La tenacidad aparente del multicapa y de la alúmina monolítica fue evaluada mediante el método de indentación de fractura [45]:

$$
\boldsymbol{n}_{\mathbf{m}}=\chi \frac{p}{\boldsymbol{c}^{g / 2}}
$$

dónde P es la carga, c la longitud de grieta y $\chi$ es igual a 0.08 para este material. Substituyendo los valores experimentales de longitud de grieta y carga se obtienen los valores de $\mathrm{K}_{\mathrm{Ic}}$ mult $=6.0 \pm 0.8 \mathrm{MPa} \mathrm{m}^{1 / 2}$ para el multicapa y $\mathrm{K}_{\mathrm{Ic}}^{\text {alum }}=3.5 \pm 0.8 \mathrm{MPa} \mathrm{m}^{1 / 2}$ para la alúmina monolítica. Se ha de tener en cuenta que estos valores de tenacidad sólo pueden tomarse como aproximados debido a las limitaciones del método. Se aprecia, sin embargo que el valor del multicapa es mucho más elevado que el de la alúmina monolítica. Esta diferencia puede atribuirse a la tensión residual $\left(\sigma_{\text {res }}\right)$ introducida por la arquitectura de la multicapa, con lo que ésta puede ser calculada mediante la relación:

$$
X_{b}=X_{h} \boldsymbol{\omega}+Y \sigma_{m} \sqrt{c_{1}}
$$

dónde $Y=1.29$. Mediante esta relación, el valor de la tensión residual se evaluó en $183 \mathrm{MPa}$ en compresión.

Los materiales se indentaron con una esfera de metal duro (WC-Co) de $2.5 \mathrm{~mm}$ de diámetro con el objeto de determinar la carga crítica para rotura Hertziana. En el caso de A/ AZ, la indentación se efectuó en la capa de alúmina superficial. Para el caso de MA, la carga crítica es $\mathrm{P}_{\mathrm{c}}^{\mathrm{A}}=607 \pm 50 \mathrm{~N}$, y para el caso de A/AZ la carga crítica es $\mathrm{P}_{\mathrm{c}}^{\mathrm{A} / \mathrm{AZ}}=675 \pm 50 \mathrm{~N}$.

A continuación, las muestras fueron sometidas a ensayos de carga constante y de carga cíclica a cargas inferiores a la carga crítica, evaluando la aparición de grieta anillo en la superficie al cabo de un determinado tiempo o número de ciclos [42]. Los ensayos con carga estática fueron efectuados en una máquina de ensayos mecánicos electromecánica Instron 8562, con una célula de carga de $5 \mathrm{kN}$, y cargas aplicadas entre 700 y $400 \mathrm{~N}$, hasta $300000 \mathrm{~s}$. Los ensayos con carga cíclica fueron efectuados en una máquina de ensayos mecánicos servohidráulica Instron 8500 con una célula de carga de $5 \mathrm{kN}$. Las cargas máximas aplicadas fueron entre 700 y $200 \mathrm{~N}$, con una carga mínima de $50 \mathrm{~N}$ para evitar deslizamientos de la muestra, a una frecuencia de $10 \mathrm{~Hz}$ y hasta 300000 ciclos. En todos los casos la esfera de WC-Co fue inspeccionada regularmente para detectar daño, y rotada o desechada en caso de que existiese deformación. Las muestras fueron sujetadas firmemente para evitar pequeños deslizamientos y aparición de fretting-fatiga.

\section{RESULTADOS Y DISCUSIÓN}

Los resultados obtenidos en carga estática se presentan en la figura 1, mientras que los obtenidos con cargas cíclicas se

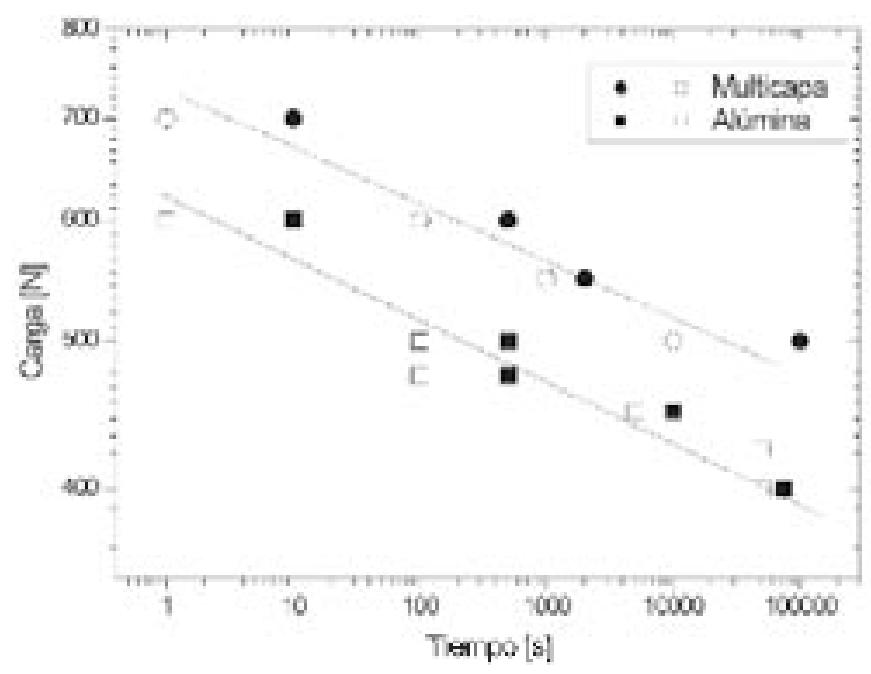

Fig. 1.- Carga aplicada frente a tiempo hasta la aparición de grieta anillo en carga estática, para A/ AZ y MA. Los símbolos blancos indican que no se aprecia daño para esa carga, mientras que los símbolos negros indican la existencia de una grieta anillo,

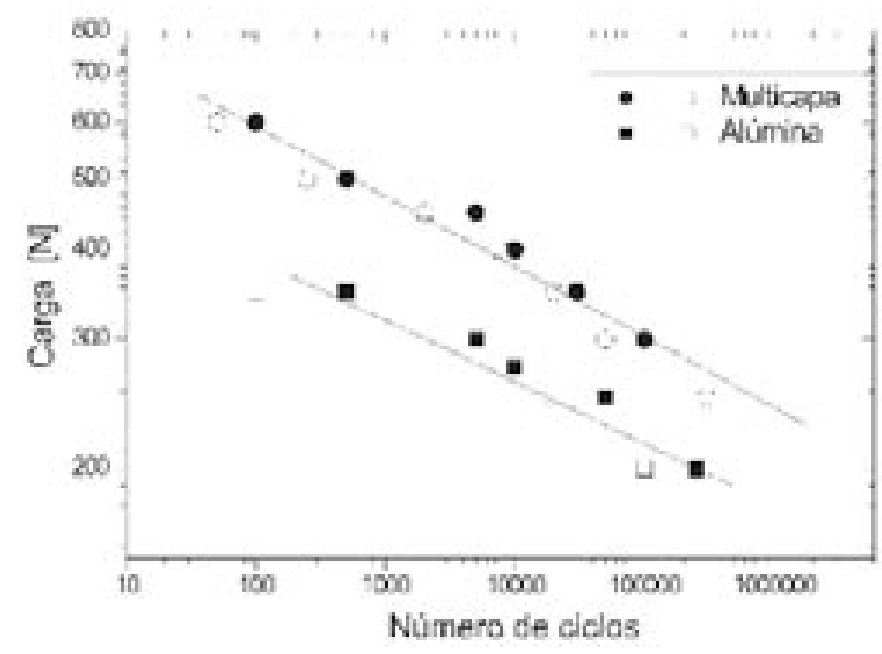

Fig. 2.- Carga aplicada frente a número de ciclos hasta la aparición de grieta anillo en carga cíclica, para A/ AZ y MA. Los símbolos blancos indican que no se aprecia daño para esa carga, mientras que los símbolos negros indican la existencia de una grieta anillo,

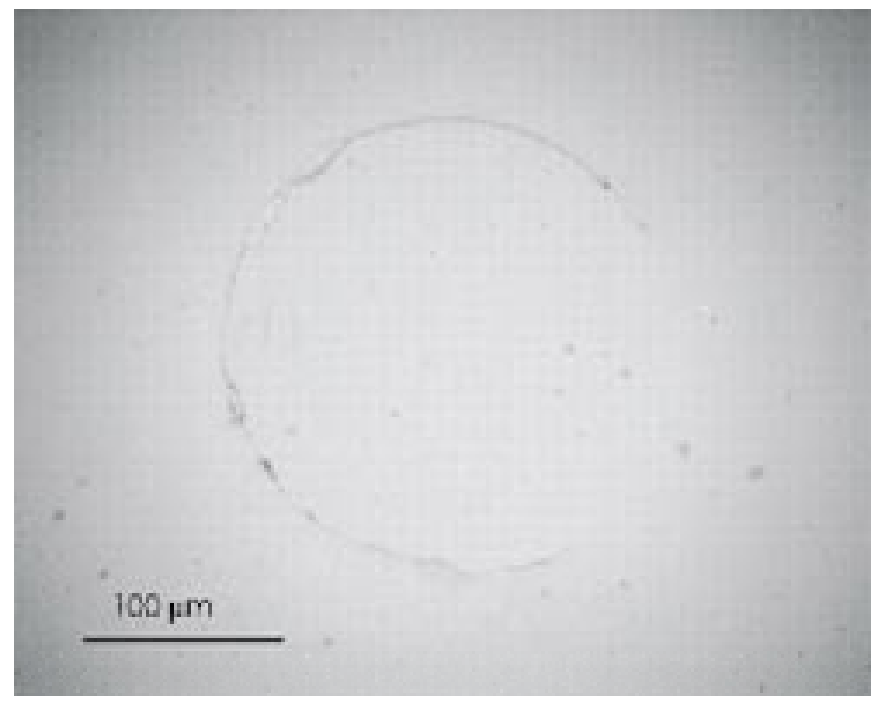

Fig. 3.- Grieta superficial producida bajo carga cíclica para un material multicapa 
presentan en la figura 2. En estas gráficas están representados los intervalos para los cuales se observa aparición de grieta anillo (ver figura 3), dónde un símbolo blanco representa la no apreciación de daño, y un símbolo negro representa la observación de una grieta anillo. En este último caso es posible que exista, aunque sea parcialmente, una pequeña grieta cono, aunque esto no es fácilmente comprobable ya que el material no es transparente.

En las figuras 1 y 2 se observa que el material A/ AZ presenta una mayor resistencia a la aparición de daño superficial que MA. Esta diferencia, que es similar para todas las cargas y tiempos, puede ser achacada a la existencia de tensiones residuales que disminuyen el factor de intensidad de tensiones efectivo, ya que en los dos materiales la microestructura es equivalente.

Comparando los comportamientos bajo cargas estáticas y cíclicas (figura 4) se aprecia que en ambos materiales aparece daño superficial antes bajo cargas cíclicas que bajo cargas estáticas. La degradación que sufren tanto MA como A/AZ puede achacarse a corrosión bajo tensión, debido a que el vapor de agua presente en la atmósfera reacciona con los enlaces cristalinos (principalmente de $\mathrm{SiO}_{2}$ ) y permite que la grieta propague subcríticamente. Cuando se somete a una carga cíclica, la corrosión bajo tensión sigue actuando cuando la grieta está suficientemente abierta como para que penetre el vapor de agua. Así, hay algunos casos en los que toda la degradación de materiales cerámicos bajo cargas cíclicas es atribuible a corrosión bajo tensión [46]. Si sólo este mecanismo está activo, entonces la degradación bajo cargas cíclicas debe ser igual o menor que bajo cargas estáticas, ya que la punta de la grieta estará sólo un breve intervalo de tiempo sometida a

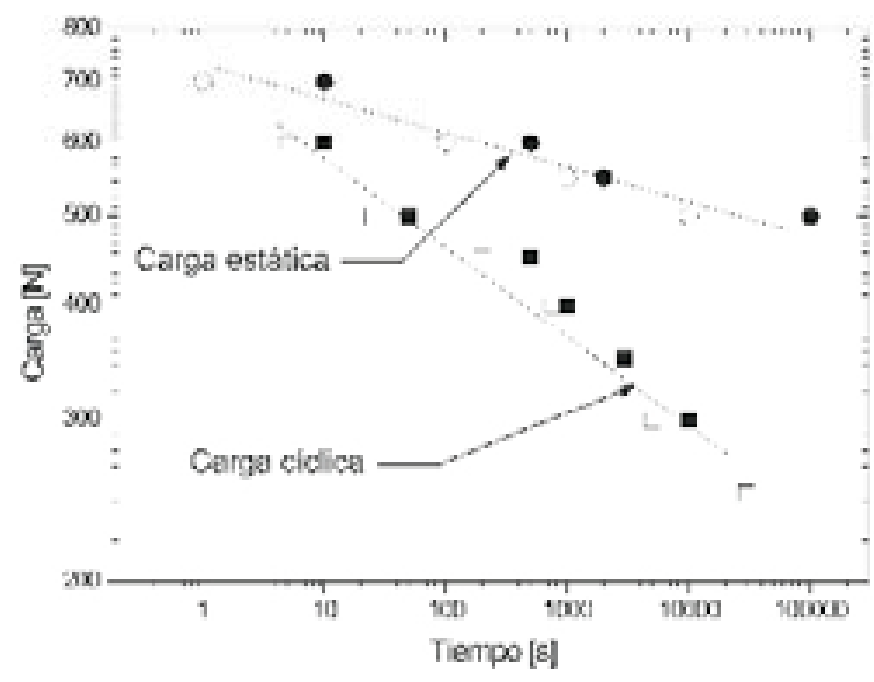

Fig. 4.- Comparación entre la aparición de grieta superficial en A/AZ para cargas estática y cíclica, en la misma escala de tiempo. Se aprecia que la carga cíclica produce daño mucho más rápidamente que la carga estática, lo que indica que existen mecanismos de fatiga y no sólo de corrosión bajo tensión

un factor de intensidad de tensiones igual al que sentiría bajo carga estática.

Sin embargo, este no es el caso, como se aprecia claramente en la figura 4. Por lo tanto, podemos llegar a la conclusión de que existe fatiga, es decir, que existe degradación de la tenacidad del material debida a cargas cíclicas. Si se observan las grietas anillo producidas mediante cargas estáticas y cíclicas en MEB, tal y como se ve en la figura 5, se aprecia que, mientras que la grieta producida con cargas estáticas es limpia, en la grieta por carga cíclica se observa que ha habido movimiento y daño en los granos adyacentes a la fractura, lo que indica la existencia de fatiga [47, 48, 49].

En el caso de carga estática, la velocidad de crecimiento subcrítico de la grieta $(\mathrm{dc} / \mathrm{dt})$ puede describirse por:

$$
\frac{d s}{d}=v_{0}\left(\frac{a_{l}}{\sigma_{2}}\right)^{n}
$$

dónde $\mathrm{v}_{0}$ es una constante del material y $\mathrm{n}$ es el exponente de crecimiento subcrítico, constante del material. El factor intensidad de tensiones total vendrá dado por la suma del factor intensidad de tensiones generado por el campo hertziano más el factor intensidad de tensiones generado por la tensión residual. Para una grieta anillo, el campo de tensiones es altamente inhomogéneo, con lo que no existen soluciones analíticas para el factor intensidad de tensiones. Sin embargo, este factor intensidad de tensiones puede aproximarse por un polinomio [50], que para profundidades pequeñas de la grieta anillo puede tomarse de orden dos:

$$
\Sigma_{I}=\sigma_{V}(\pi \omega)^{2 x}\left[f_{*}\left(\frac{c}{\sigma}\right)-f_{I}\left(\frac{c}{a}\right)\right]
$$

dónde $\mathrm{f}_{0}$ y $\mathrm{f}_{1}$ son los factores del polinomio de ajuste dependientes de la elipticidad, a es la longitud de grieta, y $\sigma_{0}$ es la tensión superficial producida por la indentación esférica e igual a [23]:

$$
\sigma_{4}=\frac{1}{2 \pi}(-2 v)\left(\frac{6 P E^{2}}{R^{2}}\right)^{1 / 4}
$$

dónde $v$ es el módulo de Poisson, P la carga aplicada, y R el radio de la esfera de indentación y $\mathrm{E}^{*}$ el módulo de Young efectivo, igual a:

$$
\frac{1}{E^{n}}=\frac{\left(1-v^{2}\right)}{E}+\frac{\left(1-v_{1}^{2}\right)}{E_{1}}
$$

dónde el subíndice i simboliza el material de la esfera de indentación.

Así, siguiendo el análisis realizado por Pavón y col. [43], la ley de crecimiento subcrítico de una grieta anillo teniendo en cuenta el factor intensidad de tensiones de la tensión residual $\left(\sigma_{\text {res }}\right)$ puede ser expresado como:

$$
\frac{d \varepsilon}{d}=\psi_{0}\left[\frac{\left.(\pi c)^{1 / 2} z(\pi / c)\left(f_{0}-f_{0}\right) \sigma_{0}+f_{0} \sigma_{n}\right]}{X_{n}}\right]^{n}
$$

dónde $\mathrm{g}(\mathrm{a} / \mathrm{c})$ es función no lineal de la elipticidad [51] y c es la longitud de grieta superficial que depende del radio de contacto $\mathrm{r}_{0} \mathrm{y}$ del ángulo que forma con el centro de la indentación $\theta$ :

$$
c=\Gamma_{b} \theta=\left(\frac{3 \pi P}{4 E^{*}}\right)^{1 / 3} \theta
$$



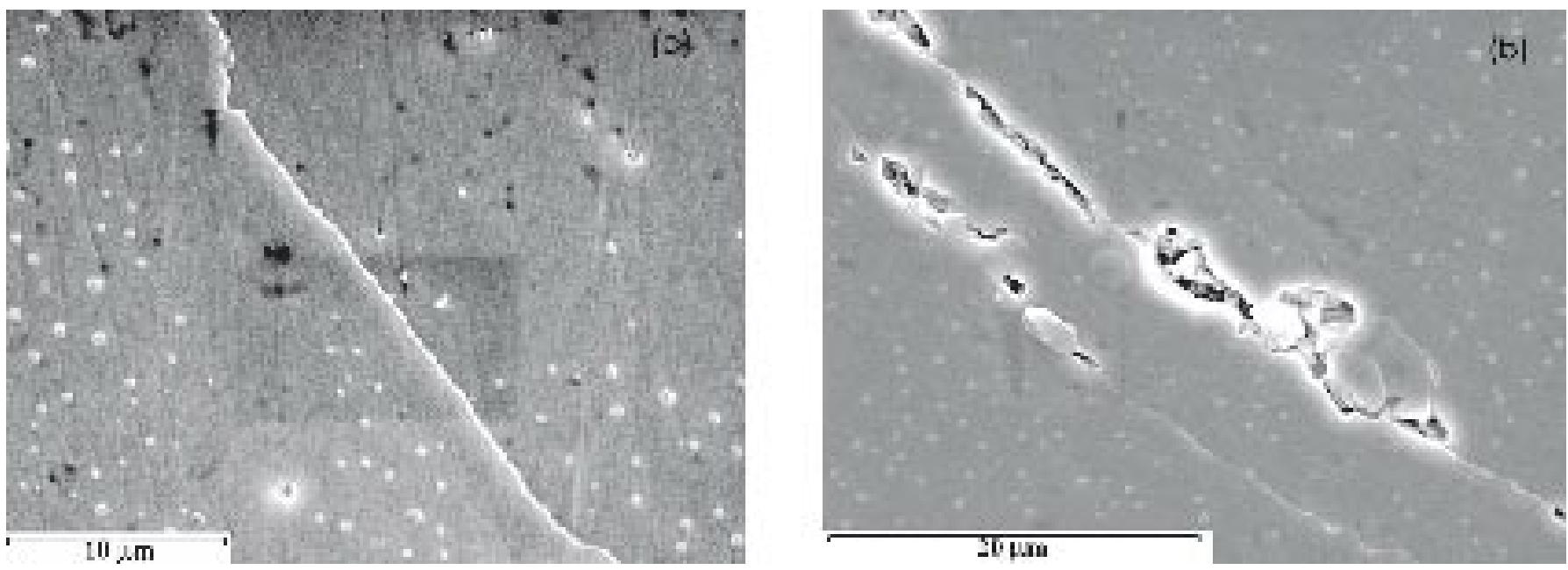

Fig. 5.- Detalle de las grietas anillo producidas bajo (a) carga estática y (b) carga cíclica en un material multicapa. Obsérvese que bajo cargas cíclicas hay degradación del material alrededor de la grieta, lo que indica existencia de fatiga.

Integrando ecuación (7) para la formación completa de grieta anillo $(\theta=2 \pi)$ y combinándola con las ecuaciones (5) y (8) es posible demostrar que:

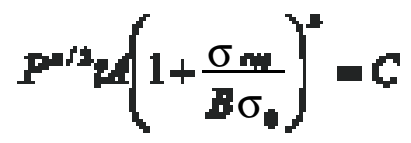

dónde $\mathrm{A}, \mathrm{B}, \mathrm{y}$ son constantes que dependen de los parámetros de ensayo [43] y t es el tiempo hasta aparición de grieta anillo. Suponiendo que la tensión superficial $\sigma_{0}$ es mayor que la tensión residual, la ecuación (9) puede aproximarse por:

$$
P^{2+2}+1=42
$$

Dónde P es la carga aplicada. En el caso de carga cíclica se puede hacer el mismo análisis partiendo de las leyes de crecimiento de grieta de Paris, llegando a una expresión equivalente a (5) pero sustituyendo t por número de ciclos. Se puede ver que como primera aproximación, la tensión residual producirá un desplazamiento en las curvas de carga frente a tiempo, mientras que la pendiente se conservará igual.

Utilizando la expresión (11) se puede calcular el valor de $\mathrm{n}$ a partir de los datos experimentales de la figura 1. Así, se obtiene un valor de $\mathrm{n}=53 \pm 9$, que es similar a los valores que se encuentran en la literatura, entre 40 [37] y 60 [52]. Análogamente, si analizamos los datos de contacto cíclico con el mismo tipo de expresión, obtenemos valores de exponentes de Paris iguales a $22 \pm 4$, similares a los obtenidos por Guiu $[47,53]$ entre 13 y 20, o los obtenidos por Nagl y col [54], iguales a 22 .

\section{CONCLUSIONES}

El material multicapa presenta una mayor resistencia a la aparición de grieta superficial producida por contacto esférico, tanto estático como cíclico, en comparación con la alúmina monolítica. Esto es debido la existencia de tensiones residuales de compresión en la superficie introducidas por la arquitectura laminar de éstos materiales. Esta tensión residual provoca que el factor de intensidad de tensiones efectivo de los materiales multicapa sea menor que el de la alúmina, retrasando, por tanto, la propagación de grieta.

Al someter tanto la alúmina como el multicapa a contacto cíclico se observa que el daño aparece antes que bajo contacto estático. Esto puede atribuirse a la aparición de fatiga por las cargas cíclicas, adicionalmente al efecto de corrosión bajo tensión que también aparece bajo cargas estáticas.

Finalmente se ha visto que las curvas experimentales de carga aplicada frente tiempo / número de ciclos, puede ser racionalizada a partir de la ley de crecimiento subcrítico o la ley de Paris utilizando las expresiones de factor intensidad de tensiones para crecimiento de grieta anillo.

\section{AGRADECIMIENTOS}

Los autores quisieran agradecer al Ministerio de Ciencia y Tecnología la ayuda recibida mediante el proyecto MAT-200200368. Work supported in part by the European Community's Human Potential Programme under contract HPRN-CT-200200203, [SICMAC].

LC and FC acknowledge the financial support provided through the European Community's Human Potential Programme under contract HPRN-CT-2002-00203, [SICMAC]. EJP agradece a la Generalitat de Catalunya la ayuda recibida a través de la beca de "Reincorporació de Doctors" RED$15 / 2002$.

\section{BIBLIOGRAFÍA}

1.- Moya, J.S. “Layered Ceramics.” Adv. Mater. 7.2 (1995): 185-189.

2.- Chan, H. M. "Layered Ceramics: proceesing and mechanical behaviour." Annu. Rev. Mater. Sci..27 (1997): 249-282.

3.- Harner, M.P. , Chan, H.M., and Miller, G. A. "Unique opportunities for microstructural engineering with duplex and laminar ceramic composites." J. Am. Ceram. Soc. 75.7 (1992): 1715-1728.

4.- Lakshminarayanan, R. , Shetty, D. K., and Cutler, R. A. . "Toughening of layered ceramic composites with residual surface compression." J. Am. Ceram. Soc. 79.11 (1996): 79-87.

5.- Tomanszewski, H., Strezeszewski, J., and Gebicki, W. "The role of residual stress in layered composites of Y-ZrO2 and Al2O3." J. Eur. Ceram. Soc. 19 (1999): 255-262. 
6.- Blattner, A.J., Lakshminarayanan, R., and Shetty, D.K. "Toughening of layered ceramic composites with residual surface compression: effect of layer thickness." Eng. Fract. Mech. 68 (2001): 1-7.

7.- Sánchez-Herencia, A.J., Pascual, C. , He, J., and Lange, F.F. "ZrO2 / ZrO2 layered composites for crack bifurcation." J. Am. Ceram. Soc. 82.6 (1999): 1512-1518.

8.- Sánchez-Herencia, A. J. , James, L., and Lange, F.F. “Bifurcaion in alumina plates produced by a phase transformation in central alumina / zirconia thin layers." J. Eur. Ceram. Soc. 20.9 (2000): 1297-1300.

9.- Moon, R.J., Hoffman, M. , Hilden, J. , Bouwman, K. , Trumble, K. , and Rödel, J. "Weigth function analysis on the R-curve behaviour of multilayered alumina-zirconia composites." J. Am. Ceram. Soc. 85.6 (2002): 1505-1511.

10.- Moon, R., Bowman, K., Trumble, K., and Rödel, J. "Comparison of R-curves from single-edge V-notched-beam (SEVNB) and Surface-Crackin-Flexure (SCF) Fracture-Toughenss test methods on Multilayer aluminazirconia composites." J. Am. Ceram. Soc. 83.2 (2000): 445-447.

11.- Hutchinson, J.W., and Suo, Z. "Mixed mode cracking in layered materials." Adv. Appl. Mech. 29 (1992): 63-191.

12.- He, M.-Y., and Hutchinson, J.W. "Crack deflection at an interface between dissimilar materials.” Int. J. Solids. Struct. 25 (1989): 1053-1067.

13.- He, M.-Y., Evans, A.G., and Hutchinson, J.W. "Crack deflection at an interface between dissimilar elastic materials: role of residual stresses." Int. J. Solids Structures 31.24 (1994): 3443-3455.

14.- Rao, M.P., Sánchez-Herencia, A.J., Beltz, G.E., McMeeking, R.M., and Lange, F.F. "Laminar Ceramics that exhibit a threshold strength." Science 286 (1999): 102-105.

15.- Rao, M.P. , Rödel, J., and Lange, F.F. "Residual Stress Induced R-curve in Laminar Ceramics that exhibit a threshold strength." J. Am. Ceram. Soc. 84.11 (2001): 2722-2724.

16.- Rao, M.P., and Lange, F.F. "Factors affecting threshold strength in laminar ceramics containing thin compressive layers." J. Am. Ceram. Soc. 85.5 (2002): 1222-1228.

17.- Ho, S. , Hillman, C., Lange, F.F., and Suo, Z. "Surface cracking in layers under biaxial, residual compressive stress." J. Am. Ceram. Soc. 78.9 (1995): 2353-2359.

18.- Lugovy, M, Orlovskaya, N. , Slyunyayev, V., Gogotsi, G., Kübler, J., and Sanchez-Herencia, A.J. "Crack bifurcation features in laminar specimens with fixed total thickness." Comp. Sci. Tech. 62 (2002): 819-830.

19.- Oechsner, M., Hillman, C., and Lange, F.F. "Crack bifurcation in laminar ceramic composites." J. Am. Ceram. Soc. 79.7 (1996): 1834-1838.

20.- Russo C.J, Harmer M.P., Chan H.M, Miller G.A. “Design of a laminated ceramic composite for improved strength and toughness" J. Am. Ceram Soc. 75.12 (1992): 3396-3400

21.- Tarlazzi, A., Roncari, E., Pinasco, P., Guicciardi, S., Melandri, C., de Portu, G. "Tribological behaviour of $\mathrm{Al}_{2} \mathrm{O}_{3} / \mathrm{ZrO}_{2}-\mathrm{Al}_{2} \mathrm{O}_{3}$ laminated composites" Wear 224 (1999): 255-262

22.- Toschi, F., Melandri, C., Pinasco, P., Roncari, E. , Guicciardi, S., and de Portu, G. "Influence of Residual Stresses on the wear behavior of alumina/ alumina-zirconia laminated composites." J. Am. Ceram. Soc. 86.9 (2003) $1547-1553$

23.- Lawn, B.R. "Indentation of ceramics with spheres: a century after Hertz." J. Am. Ceram. Soc. 81.8 (1998): 1977-1994.

24.- Rhee, Y.-W., Kim, H.-W., Deng, Y., and Lawn, B.R. "Brittle fracture versus quasi plasticity in ceramics: a simple predictive index." J. Am. Ceram. Soc. 48.3 (2001): 561-565.

25.- Lawn, B.R., Padture, N.P., Cai, H., and Giberteau, F. "Making ceramics "ductile"." Science 263 (1994): 1114-1116.

26.- Lawn BR, Deng Y, Miranda P, Pajares A, Chai H, Kim DK “Overview: Damage in brittle layer structures from concentrated loads" J. Mater. Res. 17.12 (2002): 3019-3036

27.- Miranda P, Pajares A, Guiberteau F, Deng Y, Lawn BR “Designing damageresistant brittle-coating structures: I. Bilayers" Acta Mater. 51.14 (2003): 4347-4356

28.- Miranda P, Pajares A, Guiberteau F, Deng Y, Zhao H, Lawn BR “Designing damage-resistant brittle-coating structures: II. Trilayers" Acta Mater. 51.14 (2003): 4357-4365

29.- Pajares A, Miranda P, Guiberteau F, Cumbrera FI “Mechanical characterization of materials for dental applications" Revista de Metalurgia 37.2 (2001): 316-329
30.- Miranda P, Pajares A, Guiberteau F, Cumbrera FL, Lawn BR "Role of flaw statistics in contact fracture of brittle coatings" Acta Mater. 49.18 (2001) 3719-3726

31.- An, L. , Ha, H.-C. , and Chan, H. M. "High-strength alumina/alumina: calcium-Hexaluminate layer composites." J. Am. Ceram. Soc. 81.12 (1998): 3321-3324.

32.- Jitcharoen J, Padture NP, Giannakopoulos AE, Suresh S “Hertzian-crack suppression in ceramics with elastic-modulus-graded surfaces" J. Am. Ceram. Soc. 81.9 (2001): 2301-2308

33.- An, L. "Indentation fatigue in random and textured alumina composites" J. Am. Ceram. Soc. 82.1(1999): 178-182

34.- Kim, D.K., Jung, Y.-G., Peterson, I.M., and Lawn, B.R. "Cyclic fatigue of intrinsically brittle ceramics in contact with spheres." Acta mater. 47.18 (1999): 4711-4725.

35.- Lee, K.S., Jung, Y.-G., Peterson, I.M., Lawn, B.R., Kim, D.K., and Lee, S.K. "Model for cyclic fatigue of quasi-plastic ceramics in contact with spheres." J. Am. Ceram. Soc. 83.9 (2000): 2255-2262.

36.- Lee, S.K., and Lawn, B.R. "Contact fatigue in Silicon Nitride." J. Am. Ceram. Soc. 82.5 (1999): 1281-1288.

37.- Fett, T., Keller, R., Munz. D., Ernst, E., and Thun, G. "Fatigue of alumina under contact loading." Eng. Fract. Mech. 70 (2003): 1143-1152.

38.- Fett, T., Creek, D., Badenheim, D., and Oberacker, R. "Crack growth data from dynamic tests under contact loading?." J. Eur. Ceram. Soc. 24 (2004): 2049-2054.

39.- Legin Eyzop, B., and Karlsson, S. "Cyclic contact fatigue of silicon nitride." Wear 225-229 (1999): 1303-1308.

40.- Legin Ezyop, B., and Karlsson, S. "Contact fatigue of silicon nitride." Wear 249 (2001): 208-213.

41.- Chen, Z., Cuneo, J.C., Mecholosky, J.J., and Hu, S. "Damage processes in SI3N4 bearing material under contact loading." Wear 198 (1996): 197-207.

42.- Langitan, F.B., and Lawn, B.R. "Effect of reactive enviroment on the Hertzian strength of brittle solids." J. Appl. Phys. 41.8 (1970): 3357-3365.

43.- Pavón, J.J., Jiménez-Piqué, E. , Anglada, M. , Saiz, E. , Tomsia, A. “Stress corrosion cracking of a glass coating on Ti6Al4V for biomedical applications" J. Eur. Ceram. Soc.(2005) en prensa

44.- Fiori, C., de Portu, G. "Tape Casting: a technique for preparing and studying new materials" pp. 213-25 en Proceedings of the British Ceramic Society No. 38, 1986, Novel Ceramic Fabrication Processes and Applications Editado por R.W. Davidge, Shelton, Stroke-on-Trent, Reino Unido, 1986.

45.- Anstis, G.R., Chantikul, P. and Lawn, B.R. "A critical evaluation of indentation techniques for measuring fracture toughness: I, direct crack measurements". J. Am. Ceram. Soc. 64 (1981):533-538

46.- Roebben, G., Steen, M., Bressers, J., and van der Biest, O. "Mechanical fatigue in monolithic non-transforming ceramics." Prog. Mat. Sci. 40 (1996) 265-331.

47.- Li, M., and Guiu, F.; "Subcritical crack growth in alumina- I. Effects of grain size, specimen size and loading mode." Acta metall. mater. 43.5 (1995): 1859-1869.

48.- Li, M., and Guiu, F. "Subcritical crack growth in alumina- II. Crack bridging and cyclic fatigue mechanisms." Acta metall. mater. 43.5 (1995): 1871-1884.

49.- Kruzic, J.J., Cannon, R.M., and Ritchie, R.O. “Crack-size effects on cyclic and monotonic crack growth in polycristaline alumina: quantification of the role of grain bridging." J. Am. Ceram. Soc. 87.1 (2004): 93-103

50.- Licht, V., Hüselmeier, P., and Fett, T. "Probability of cone crack initation due to spherical indentation due to spherical contact loading." J. Eur. Ceram. Soc. 24 (2004): 2907-2915.

51.- Warren, P.D. “Determining the Fracture Toughness of Brittle Materials by Hertzian Indentation." J. Eur. Ceram. Soc. 15 (1995): 201-207.

52- Barinov, S.M. Ivanov, N.V., Orlov, S.V., and Shevchenko, V.J. "Influence of Enviroment on delayed failure of alumina ceramics." J. Eur. Ceram. Soc. 18 (1998): 2057-2063.

53.- Guiu, F., Reece, M.J., and Vaughan, D.A.J. "Cyclic fatigue of ceramics.” J. Mater. Sci. 26 (1991): 3275-3286.

54.- Nagl, M.M.; Llanes, L.; Fernández, R. y Anglada, M. “The fatigue behaviour of Mg-PSZ and ZTA Ceramics". En Fracture Mechanics of Ceramics Vol 12, editado por Bradt R.C., Plenum Press, Nueva York (EEUU), 1996 